MCTP-03-44 / UT-03-33

\title{
Neutrino-induced lepton flavor violation in gauge-mediated supersymmetry breaking
}

\author{
Kazuhiro Tobe $^{a, b}$, James D. Wells ${ }^{a}$, Tsutomu Yanagida ${ }^{c}$ \\ (a) Michigan Center for Theoretical Physics (MCTP) \\ University of Michigan, Ann Arbor, MI 48109-1120, USA \\ ${ }^{(b)}$ Department of Physics, University of California, Davis 95616, USA \\ (c) Department of Physics, University of Tokyo, Tokyo 113-0033, Japan
}

\begin{abstract}
Gauge-mediated supersymmetry breaking is known to greatly suppress flavor changing neutral current effects. However, we show that gauge mediation in the context of leptogenesis implies potentially large lepton flavor violating signals. If the heavy righthanded neutrinos that participate in leptogenesis are lighter than the messenger scale of gauge mediation, they will induce flavor off-diagonal masses to the sleptons which in turn can induce large effects in $\mu \rightarrow e \gamma, \tau \rightarrow \mu \gamma$, and $\mu-e$ conversion in nuclei. We demonstrate this result and compute numerically the lepton-flavor violating decay and conversion rates in scenarios of direct gauge mediation.
\end{abstract}

hep-ph/0310148

October 2003 


\section{Introduction}

One of the challenges of supersymmetry is constructing a theory that has no new sources of flavor violation substantially beyond those already present in the Standard Model (SM). This requirement is thrust upon us by experiment, yet there are theory ideas that predict it.

One such flavor-tame theory is gauge mediated supersymmetry breaking (GMSB). In minimal models [1, 2] of GMSB (mGMSB) there are $n_{m}$ copies of $5+\overline{5}$ messengers that feel supersymmetry breaking via a gauge singlet, $S=M+\theta^{2} F_{S}$, in which $M$ is a messenger mass and $F_{S}$ represents a supersymmetry breaking scale in the messenger sector. Since the messenger particles interact with minimal supersymmetric SM (MSSM) particles only through the SM gauge forces, the messenger-loop diagrams give rise to flavor-diagonal supersymmetry breaking masses in the MSSM sector at the messenger scale $M$, and hence flavor violation in supersymmetry breaking masses are automatically suppressed at the messenger scale $M[1]$.

The messenger scale can be any numerical value in principle. From a collider physics standpoint it is very interesting when $M$ and $F$ (goldstino auxiliary component) are as low as possible as it can potentially lead to prompt decays of the lightest SM superpartner into its corresponding SM particle plus gravitino [2]. The collider signatures for this are usually easy to distinguish from background and if found would provide a compelling case for gauge mediation or some close variant of the theory.

Despite the interesting collider phenomenology of low-scale messengers, there are some advantages to considering a much higher messenger scale. This is particularly true when we consider how massive neutrinos fit into the full theory, as nowadays results from neutrino oscillation experiments clearly require. These neutrinos are best described by a seesaw mechanism with very heavy right-handed neutrinos, $N_{i}[3]$. Out of equilibrium decays of these heavy neutrinos can precipitate leptogenesis, leading to proper baryogenesis through sphaleron reprocessing [4. Applying all these criteria we expect the right-handed neutrino masses to lie somewhere in the range [5, 6]

$$
10^{6-8} \mathrm{GeV} \lesssim M_{R_{i}} \lesssim 10^{12-16} \mathrm{GeV}
$$

Making leptogenesis work requires that the reheat temperature be at least as large as $T_{R}>10^{6} \mathrm{GeV}$ [5, 6]. Gravitinos can be produced copiously during the reheat phase of the universe [7]. For any given reheat temperature there is a lower bound on the mass of the 
gravitino such that the universe does not become overclosed. Near this overclosure limit, the gravitino can be a significant component of the dark matter. For $T_{R}=10^{6} \mathrm{GeV}$ that lower limit is $m_{3 / 2}>100 \mathrm{MeV} .{ }^{1}$ Thus, supergravity tells us

$$
m_{3 / 2}=\frac{F}{\sqrt{3} M_{\mathrm{Pl}}} \gtrsim 100 \mathrm{MeV} \Longrightarrow \sqrt{F} \gtrsim \text { few } \times 10^{8} \mathrm{GeV}
$$

Keeping flavor-mixing supergravity contributions to scalar masses sufficiently suppressed is equivalent to requiring $m_{3 / 2} \lesssim 10 \mathrm{GeV}$. Therefore, we have a lower limit and an upper limit for $F$,

$$
10^{17} \mathrm{GeV}^{2} \lesssim F \lesssim 10^{19} \mathrm{GeV}^{2} \quad \text { (allowed range for } F \text { ) }
$$

The supersymmetry breaking $F_{S}$-term(s) that participate in GMSB are not necessarily the full strength $F$-term(s) that add to the gravitino mass. In the early Dine-Nelson GMSB models [1], $F_{S}$ was a loop factor below the $F$ in Eq. (2). However, there is a class of GMSB models, which we call direct gauge mediation, where $F_{S}=F$ [9, 10, 11, 12, 13, 14]. Combining the collider limits on the gaugino masses $M_{i} \gtrsim 100 \mathrm{GeV}$ with the allowed range for $F$ obtained from requirements on the gravitino mass in direct gauge mediation, we can estimate the allowed range for the messenger mass,

$$
10^{12} \mathrm{GeV} \lesssim M \lesssim 10^{15} \mathrm{GeV} \quad \text { (messenger mass range) }
$$

Interestingly successful leptogenesis induced by heavy right-handed neutrinos leads to high messenger scale $M$ in direct gauge mediation.

Under the requirements in Eqs. (3), 4), the phenomenology no longer admits prompt decays of the lightest superpartner into a gravitino. If the lightest SM superpartner is a neutralino the phenomenology will be hard to distinguish from a regular supergravity model. ${ }^{2}$ However, the interesting phenomenology of these models does not stop with collider physics. Indeed, in GMSB with such a high-messenger scale, heavy right-handed neutrinos play an important role in low-energy phenomenology as well as in leptogenesis. Perhaps the most important and unique phenomenological implication of this theory is the potentially large lepton flavor violating (LFV) signals that can arise. This is counter-intuitive since GMSB

\footnotetext{
${ }^{1}$ If there is an entropy production after the freeze-out time of the gravitino, this limit would not be applicable because it dilutes the number density of the gravitino 8 .

${ }^{2}$ A charged lightest SM superpartner that is long-lived on collider time scales would be assumed to be metastable since copious charged dark matter is not allowed cosmologically. Therefore, one would conclude that it must decay to something else, and a light gravitino would be the leading candidate for a decay product that carries away odd R-parity.
} 
is usually thought to not allow superpartner-induced LFV amplitudes. In this article we demonstrate why LFV happens in this general class of supersymmetry theories, and compute the expected rates for LFV observables $B(\mu \rightarrow e \gamma), B(\tau \rightarrow \mu \gamma)$, and $\mu-e$ conversion in nuclei. ${ }^{3}$

\section{Supersymmetric SM with right-handed neutrinos}

We begin by reviewing the MSSM with right-handed neutrinos, in which the seesaw mechanism naturally induces tiny neutrino masses. The superpotential in the lepton sector is given by

$$
W=y_{e}^{i} E_{i} L_{i} H_{1}+y_{\nu}^{i, j} N_{i} L_{j} H_{2}+\frac{M_{R_{i}}}{2} N_{i} N_{i}
$$

Here we take a basis where the charged lepton Yukawa matrix $y_{e}$ and the right-handed neutrino mass matrix $M_{R}$ are both diagonal. Note that in general the neutrino Yukawa couplings $y_{\nu}$ are not diagonal and become a source for LFV phenomena such as neutrino oscillation, $\mu \rightarrow e \gamma$, etc.

Below each right-handed neutrino mass scale $M_{R_{i}}$, the corresponding right-handed neutrino $N_{i}$ decouples. We write the resulting low-energy effective superpotential for the neutrino masses at the electroweak scale as follows,

$$
\begin{aligned}
W_{\mathrm{eff}} & =-\frac{\kappa_{i j}}{2}\left(L_{i} H_{2}\right)\left(L_{j} H_{2}\right), \\
\kappa_{i j} & \equiv\left(y_{\nu}^{\mathrm{T}} M_{R}^{-1} y_{\nu}\right)_{i j} .
\end{aligned}
$$

The neutrino mass matrix then is given by

$$
m_{\nu}=\kappa\left\langle H_{2}^{0}\right\rangle^{2}=\frac{\kappa v^{2} \sin ^{2} \beta}{2},
$$

which can be diagonalized by the Maki-Nakagawa-Sakata (MNS) matrix $U^{\mathrm{MNS}}$ [16]:

$$
\begin{array}{r}
\left(U^{\mathrm{MNS}}\right)^{\mathrm{T}} m_{\nu} U^{\mathrm{MNS}}=\operatorname{diag}\left(m_{\nu_{1}}, m_{\nu_{2}}, m_{\nu_{3}}\right), \\
\left(U^{\mathrm{MNS}}\right)^{\mathrm{T}} \kappa U^{\mathrm{MNS}}=\operatorname{diag}\left(\kappa_{1}^{D}, \kappa_{2}^{D}, \kappa_{3}^{D}\right) .
\end{array}
$$

From Eqs. (17) and (10), the neutrino Yukawa matrix can be expressed as [17,

$$
y_{\nu}^{i j}=\sqrt{M_{R_{i}}} R_{i k} \sqrt{\kappa_{k}^{D}}\left(U^{\mathrm{MNS} *}\right)_{j k}
$$

\footnotetext{
${ }^{3}$ Within the framework of supergravity supersymmetry breaking, leptogenesis and LFV have been discussed in Ref. [15].
} 
where the matrix $R$ is an unknown orthogonal matrix $R^{\mathrm{T}} R=\mathbf{1}$. Low-energy neutrino oscillation experiments determine the neutrino mass differences and the entries of the MNS matrix. Here we parameterize the MNS matrix as follows:

$$
U^{\mathrm{MNS}}=\left(\begin{array}{ccc}
c_{12} c_{13} & s_{12} c_{13} & s_{13} e^{-i \delta} \\
-s_{12} c_{23}-c_{12} s_{23} s_{13} e^{i \delta} & c_{12} c_{23}-s_{12} s_{23} s_{13} e^{i \delta} & s_{23} c_{13} e^{i \delta} \\
s_{12} s_{23}-c_{12} c_{23} s_{13} e^{i \delta} & -c_{12} s_{23}-s_{12} c_{23} s_{12} e^{i \delta} & c_{23} c_{13}
\end{array}\right)
$$

where $s_{i j}=\sin \theta_{i j}$ and $c_{i j}=\cos \theta_{i j}$.

In our analysis, we adopt the following numerical data [18, 19]:

$$
\begin{aligned}
\sin ^{2} 2 \theta_{23} & =1 \\
\Delta m_{\mathrm{atm} .}^{2} & =m_{\nu_{3}}^{2}-m_{\nu_{2}}^{2}=2 \times 10^{-3} \mathrm{eV}^{2}
\end{aligned}
$$

for atmospheric neutrinos, and

$$
\begin{aligned}
\theta_{12} & =32.5 \text { degrees } \\
\Delta m_{\text {solar }}^{2} & =m_{\nu_{2}}^{2}-m_{\nu_{1}}^{2}=7.1 \times 10^{-5} \mathrm{eV}^{2}
\end{aligned}
$$

for solar neutrinos. We assume hierarchical neutrino masses, so that we take $m_{\nu_{1}}=0$ in our analysis. Angle $\theta_{13}$ has not been determined yet, and its experimental limit is $\left|s_{13}\right| \leq 0.2$ [18]. In our numerical analysis, we will take $s_{13}=0$ or 0.1 as reference values. For simplicity, we neglect complex phase $\delta$.

\section{Lepton flavor violation in GMSB}

Off-diagonal components in the neutrino Yukawa couplings give rise to LFV neutrino oscillations, and they also can induce significant LFV in the charged lepton sector. For example, even if the supersymmetry breaking mechanism is flavor-blind, as is the case with GMSB, LFV in the slepton masses can be induced through radiative corrections (i.e., renormalization group running) involving neutrino Yukawa interactions [20, 21]. However, effects of neutrino Yukawa couplings decouple if the scale of supersymmetry breaking is lower than the righthanded neutrino mass scales. In that case, the LFV in the slepton mass matrix is negligibly small. Low-energy GMSB behaves in this manner if the messenger scale is less than the right-handed neutrino masses.

However, in the case of high-scale direct gauge mediation, which we motivated in the introduction, the messenger scale where supersymmetry breaking terms are induced can be 
higher than the right-handed neutrino mass scales, $M>M_{R_{i}}$. In this case, the neutrino Yukawa couplings enter the slepton mass matrix renormalization group equation over a significant scale range, thereby affecting potentially large LFV in the charged lepton sector. ${ }^{4}$

We can see this LFV effect through the renormalization group equations (RGEs) for the left-handed slepton masses:

$$
\begin{aligned}
\mu \frac{d}{d \mu}\left(m_{\tilde{L}}^{2}\right)_{i j} & =\left.\mu \frac{d}{d \mu}\left(m_{\tilde{L}}^{2}\right)_{i j}\right|_{\mathrm{MSSM}} \\
& +\frac{1}{16 \pi^{2}}\left[m_{\tilde{L}}^{2} y_{\nu}^{\dagger} y_{\nu}+y_{\nu}^{\dagger} y_{\nu} m_{\tilde{L}}^{2}+2\left(y_{\nu}^{\dagger} m_{\tilde{\nu}}^{2} y_{\nu}+m_{H_{2}}^{2} y_{\nu}^{\dagger} y_{\nu}+A_{\nu}^{\dagger} A_{\nu}\right)\right]_{i j},
\end{aligned}
$$

where $\mu d\left(m_{\tilde{L}}^{2}\right)_{i j} /\left.d \mu\right|_{\text {MSSM }}$ is a contribution from MSSM without right-handed neutrinos. Even if $m_{\tilde{L}}^{2}$ and $m_{\tilde{\nu}}^{2}$ are flavor-diagonal at the messenger scale, flavor mixings in the neutrino Yukawa couplings generate flavor violation in the left-handed slepton masses $m_{\tilde{L}}^{2}$. The flavor mixing in the left-handed slepton masses is approximately proportional to $y_{\nu}^{\dagger} y_{\nu}$. Using Eq. (11),

$$
\begin{aligned}
\left(y_{\nu}^{\dagger} y_{\nu}\right)_{\mu e}= & \left(U^{\mathrm{MNS}} \sqrt{\kappa^{D}} R^{\dagger} M_{R} R \sqrt{\kappa^{D}} U^{\mathrm{MNS} \dagger}\right)_{\mu e}, \\
\simeq & U_{\mu 3}^{\mathrm{MNS}} U_{e 3}^{\mathrm{MNS} *} \kappa_{3}^{D} M_{R_{3}}\left(\left|R_{33}\right|^{2}+\frac{M_{R_{2}}}{M_{R_{3}}}\left|R_{23}\right|^{2}+\frac{M_{R_{1}}}{M_{R_{3}}}\left|R_{13}\right|^{2}\right) \\
& +U_{\mu 3}^{\mathrm{MNS}} U_{e 2}^{\mathrm{MNS} *} \sqrt{\kappa_{3}^{D} \kappa_{2}^{D}} M_{R_{3}}\left(R_{33}^{*} R_{32}+\frac{M_{R_{2}}}{M_{R_{3}}} R_{23}^{*} R_{22}+\frac{M_{R_{1}}}{M_{R_{3}}} R_{13}^{*} R_{12}\right) \\
& +U_{\mu 2}^{\mathrm{MNS}} U_{e 3}^{\mathrm{MNS} *} \sqrt{\kappa_{2}^{D} \kappa_{3}^{D}} M_{R_{3}}\left(R_{32}^{*} R_{33}+\frac{M_{R_{2}}}{M_{R_{3}}} R_{22}^{*} R_{23}+\frac{M_{R_{1}}}{M_{R_{3}}} R_{12}^{*} R_{13}\right) \\
& +U_{\mu 2}^{\mathrm{MNS}} U_{e 2}^{\mathrm{MNS} *} \kappa_{2}^{D} M_{R_{3}}\left(\left|R_{32}\right|^{2}+\frac{M_{R_{2}}}{M_{R_{3}}}\left|R_{22}\right|^{2}+\frac{M_{R_{1}}}{M_{R_{3}}}\left|R_{12}\right|^{2}\right) .
\end{aligned}
$$

In Eq. (19), we assumed $\kappa_{1}^{D} \ll \kappa_{2,3}^{D}$.

For $\tau \rightarrow \mu$ flavor violation, indices $\mu$ and $e$ should be replaced by $\tau$ and $\mu$, respectively. As can be seen from Eq. (19), LFV is induced by flavor mixings from $U^{\mathrm{MNS}}$ and $R$. Although the matrix $R$ is not known, the large neutrino mixing angles in $U^{\mathrm{MNS}}$ suggested by the atmospheric and solar neutrino experiments imply that large LFV effects are possible when at least one $M_{R_{i}}<M .^{5}$

\footnotetext{
${ }^{4}$ Since the messenger scale $M$ is lower than the GUT scale, GUT-induced LFV in superpartner masses is absent.

${ }^{5}$ The hierarchical neutrino Yukawa couplings also induce non-degeneracy in diagonal components of the left-handed slepton mass matrix. Therefore, the precise measurement of slepton and sneutrino masses also would be important to probe the effects of the neutrino Yukawa couplings.
} 
In this article we mainly adopt mGMSB to calculate event rates of LFV processes. In mGMSB, MSSM gaugino $\left(M_{i}, i=1-3\right)$ and sfermion masses are generated at the messenger scale $M$ as follows:

$$
\begin{aligned}
M_{i}(M) & =n_{m} \frac{\alpha_{i}(M)}{4 \pi} \frac{F_{S}}{M} \\
\tilde{m}^{2}(M) & =\frac{n_{m}}{8 \pi^{2}}\left\{c_{1} \alpha_{1}^{2}(M)+c_{2} \alpha_{2}^{2}(M)+c_{3} \alpha_{3}^{2}(M)\right\} \frac{F_{S}^{2}}{M^{2}}
\end{aligned}
$$

where $\alpha_{i}$ are $S U(5)$-GUT normalized gauge couplings. The $c_{i}$ are the Casimirs of the sparticle representations under the SM gauge groups, which in the SM translates to

$$
c_{1}=\frac{3}{5}\left(\frac{Y}{2}\right)^{2}, \quad c_{2}=\left\{\begin{array}{c}
3 / 4, \text { if } \mathrm{SU}(2) \text { doublet } \\
0, \text { if } \mathrm{SU}(2) \text { singlet }
\end{array}, \quad c_{3}=\left\{\begin{array}{c}
4 / 3, \text { if } S U(3) \text { triplet } \\
0, \text { if } S U(3) \text { singlet }
\end{array}\right.\right.
$$

Below the messenger scale $M$, we consider the MSSM with massive right-handed neutrino(s) as the effective theory. We then numerically compute RGEs from the messenger scale $M$ to electroweak scale, taking into account decoupling of the right-handed neutrino $N_{i}$ at each mass scale $M_{R_{i}}$. During the RG running between $M$ and $M_{R_{i}}$, neutrino Yukawa interactions generate LFV in the slepton masses as shown in Eq. (17), and as a result, low-energy LFV events are induced.

In order to show the messenger-model-dependence of the result, we also consider the $Q-S-\phi$ model of Ref. [13] as an example that has a complicated messenger sector. ${ }^{6}$ In this model, at the highest energies the gauge group is $S U(5)_{H} \times S U(5)_{G}$ with gauge couplings $\alpha_{H}$ and $\alpha_{G}$ respectively. We assume all SM matter fields couple only to $S U(5)_{H}$. Below the threshold of the $Q$ states $M_{Q}$, the gauge group breaks to $S U(5)_{G} \times S U(5)_{H} \rightarrow S U(5)$ with resulting gauge coupling $\alpha$. The low-energy superpartner spectrum at the scale $M_{Q}$ can be computed using the techniques of Ref. [22]:

$$
\begin{aligned}
& M_{i}\left(M_{Q}\right)=\frac{\alpha\left(M_{Q}\right)}{4 \pi} \frac{F}{M_{Q}}\left(b_{S}-b_{H}-b_{G}\right) \\
& \tilde{m}^{2}\left(M_{Q}\right)=\frac{c \alpha^{2}\left(M_{Q}\right)}{8 \pi^{2}} \frac{F^{2}}{M_{Q}^{2}}\left\{b_{H} \frac{\alpha_{H}^{2}\left(M_{Q}\right)}{\alpha^{2}\left(M_{Q}\right)}+b_{S}-2\left(b_{H}+b_{G}\right)\right\} .
\end{aligned}
$$

where $c$ is defined in Eq. (22), and the $b_{i}$ are defined to be the coefficients of the gauge $\beta$ functions (see Eq. (5) and just below Eq. (84) of Ref. [22] for explanation of these values). In our numerical analysis, we will assume $\alpha_{H}^{2}\left(M_{Q}\right) / \alpha^{2}\left(M_{Q}\right)=1$ for simplicity. ${ }^{7}$ It is necessary

\footnotetext{
${ }^{6}$ We call the model of Ref. [13] the " $Q$ - $S$ - $\phi$ model" because it contains three messenger mass scales $M_{Q}$, $M_{S}$ and $M_{\phi}$ associated with $Q, S$ and $\phi$ states, where $M_{Q}=\lambda v_{Q} / \sqrt{5}\left(\sim 10^{15} \mathrm{GeV}\right), M_{S}=\left(v_{Q} / \sqrt{5}\right)^{3} / M_{\mathrm{Pl}}^{2}(\sim$ $\left.10^{8} \mathrm{GeV}\right)$ and $M_{\phi}=\left(v_{Q} / \sqrt{5}\right)^{4} / M_{\mathrm{Pl}}\left(\sim 10^{5} \mathrm{GeV}\right)$.

${ }^{7}$ In the case with $\alpha_{H}^{2}\left(M_{Q}\right) / \alpha^{2}\left(M_{Q}\right)=1, A$-terms vanish at $M_{Q}$.
} 
to apply the above scalar mass equation for each gauge group the superpartner scalar is charged under, and then sum the result to obtain the mass. At thresholds $M_{S}$ and $M_{\phi}$ where chiral messenger fields $S$ and $\phi$ decouple respectively, additional gaugino and scalar masses are generated. As we want to fully incorporate Yukawa coupling effects, we treat Eq. (24) as a boundary condition at the scale $M_{Q}$ and numerically evolve the masses to the weak scale across the $S$-fields and $\phi$-fields thresholds. We refer the reader to Ref. [13] for a detailed description of these thresholds.

In the subsequent sections, we will investigate this LFV phenomena employing several plausible assumptions about the mixing angles and masses of the neutrino sector. We will find that the LFV observables $\mu \rightarrow e \gamma, \mu-e$ conversion in nuclei and $\tau \rightarrow \mu \gamma$ can grow significantly above the SM predictions in several interesting cases, and therefore the ideas can be tested by present experiment and future upgrades of these LFV experiments.

\subsection{Degenerate right-handed neutrinos $M_{R_{3}}=M_{R_{2}}=M_{R_{3}}$}

First we consider a case in which all right-handed neutrinos are degenerate $M_{R_{3}}=M_{R_{2}}=$ $M_{R_{3}} \equiv M_{R}$. In this case, Eq. (19) is written by

$$
\left(y_{\nu}^{\dagger} y_{\nu}\right)_{\mu e} \simeq U_{\mu 3}^{\mathrm{MNS}} U_{e 3 *}^{\mathrm{MNS}} \kappa_{3}^{D} M_{R_{3}}+U_{\mu 2}^{\mathrm{MNS}} U_{e 2 *}^{\mathrm{MNS}} \kappa_{2}^{D} M_{R_{2}}
$$

Note that there is no $R$ matrix dependence in this case. Therefore if neutrino masses, mixings and superpartner masses are fixed, LFV event rates are expressed as a function of the messenger scale $M$ and the right-handed neutrino mass scale $M_{R}$. In Fig. 1, the $\mu-e$ conversion rate $R(\mu \rightarrow e$ in $\mathrm{Ti})$ is shown as a function of $M$ and $M_{R}$. Here we take $U_{e 3}^{\mathrm{MNS}}=0$ and $\tan \beta=30$. We assume mGMSB with $n_{m}=1$ and wino mass $\left(M_{2}\right)$ to be $200 \mathrm{GeV}$, which fixes the entire superpartner mass spectrum. As can be seen from Fig. 1, as $M_{R}$ becomes larger, the $\mu-e$ conversion rate gets larger as long as the messenger scale $M$ is larger than, and not close to, $M_{R}$. As can be understood from Eq. (25), the third generation right-handed neutrino does not contribute to $\mu-e$ flavor violating slepton masses when $U_{e 3}^{\mathrm{MNS}}=0$. When $U_{e 3}^{\mathrm{MNS}}=O(0.1)$, the third generation contribution to $\mu-e \mathrm{LFV}$ in slepton masses becomes dominant, and hence the $\mu-e$ conversion rate gets larger in general than what we present in Fig. 1. The effect of non-zero $U_{e 3}^{\mathrm{MNS}}$ would be more important in some case with non-degenerate right-handed neutrinos as we will discuss later.

In the supersymmetric SM with right-handed neutrinos, the magnetic-moment-type photon penguin diagram, which also induces the $\mu \rightarrow e \gamma$ process, is almost always dominant in 


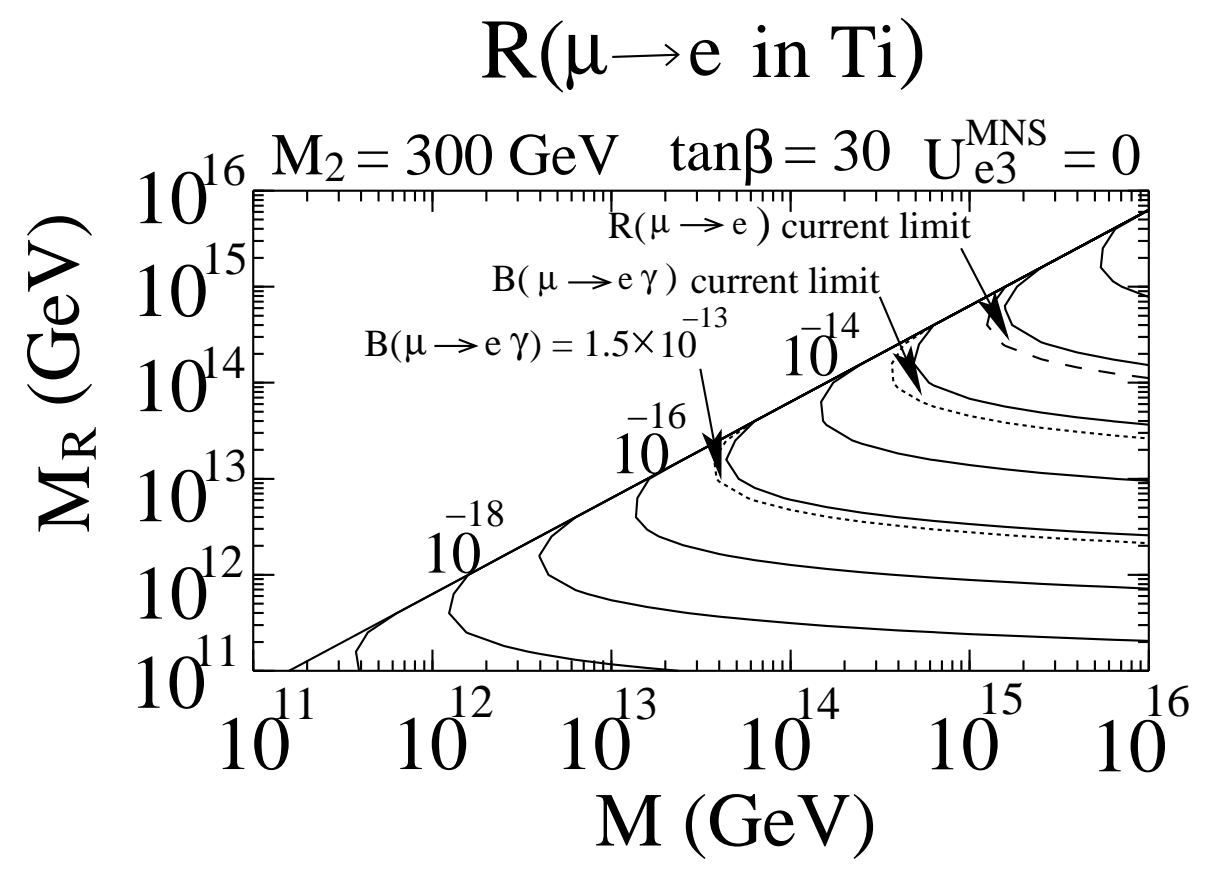

Figure 1: The $\mu-e$ conversion rates $R(\mu \rightarrow e$ in Ti) as a function of the messenger scale $M$ and the degenerate right-handed neutrino scale $M_{R}$. Here we take $U_{e 3}^{\mathrm{MNS}}=0$ and $\tan \beta=30$. We assume mGMSB with $n_{m}=1$. The low-energy wino mass $\left(M_{2}\right)$ is set to be $200 \mathrm{GeV}$, which fixes the entire superpartner mass spectrum. Current limits on $R(\mu \rightarrow e$ in Ti) and $B(\mu \rightarrow e \gamma)$, and a future sensitivity at MEG $\left[B(\mu \rightarrow e \gamma)=1.5 \times 10^{-13}\right]$ are also shown. Future $\mu-e$ conversion experiments may reach a sensitivity close to $R(\mu-e) \simeq 10^{-18}$. Note, there is no LFV above the diagonal line as $M_{R}$ is above the messenger scale.

the $\mu-e$ conversion process, and hence there is a relation between the predicted $B(\mu \rightarrow e \gamma)$ and $R(\mu \rightarrow e$ in $\mathrm{Ti})$ :

$$
\frac{R(\mu \rightarrow e \text { in } \mathrm{Ti})}{B(\mu \rightarrow e \gamma)} \simeq 5 \times 10^{-3} .
$$

This relationship is what allows us to show in Fig. 1 1 simultaneously the current limits from $\mu-e$ conversion process $\left[R(\mu \rightarrow e\right.$ in Ti $\left.)=6.1 \times 10^{-13}\right]$ [25], $\mu \rightarrow e \gamma$ process $[B(\mu \rightarrow$ $\left.e \gamma)=1.2 \times 10^{-11}\right]$ [25], and a future sensitivity at MEG experiment $[B(\mu \rightarrow e \gamma)=1.5 \times$ $\left.10^{-13}\right]$ [25, 24]. We also note that the expected sensitivity at the future MECO experiment for $\mu-e$ conversion will be $R(\mu \rightarrow e)=10^{-16}$ [25, 26], and further future experiment may be able to reach a sensitivity $R(\mu \rightarrow e)=10^{-18}$ according to studies in Ref. [27]. Therefore, at present, the current $\mu \rightarrow e \gamma$ limit already constrains the region $M_{R}>10^{14} \mathrm{GeV}$ and $M>10^{15} \mathrm{GeV}$ in Fig. 1. The future MEG and MECO experiments will be sensitive to 


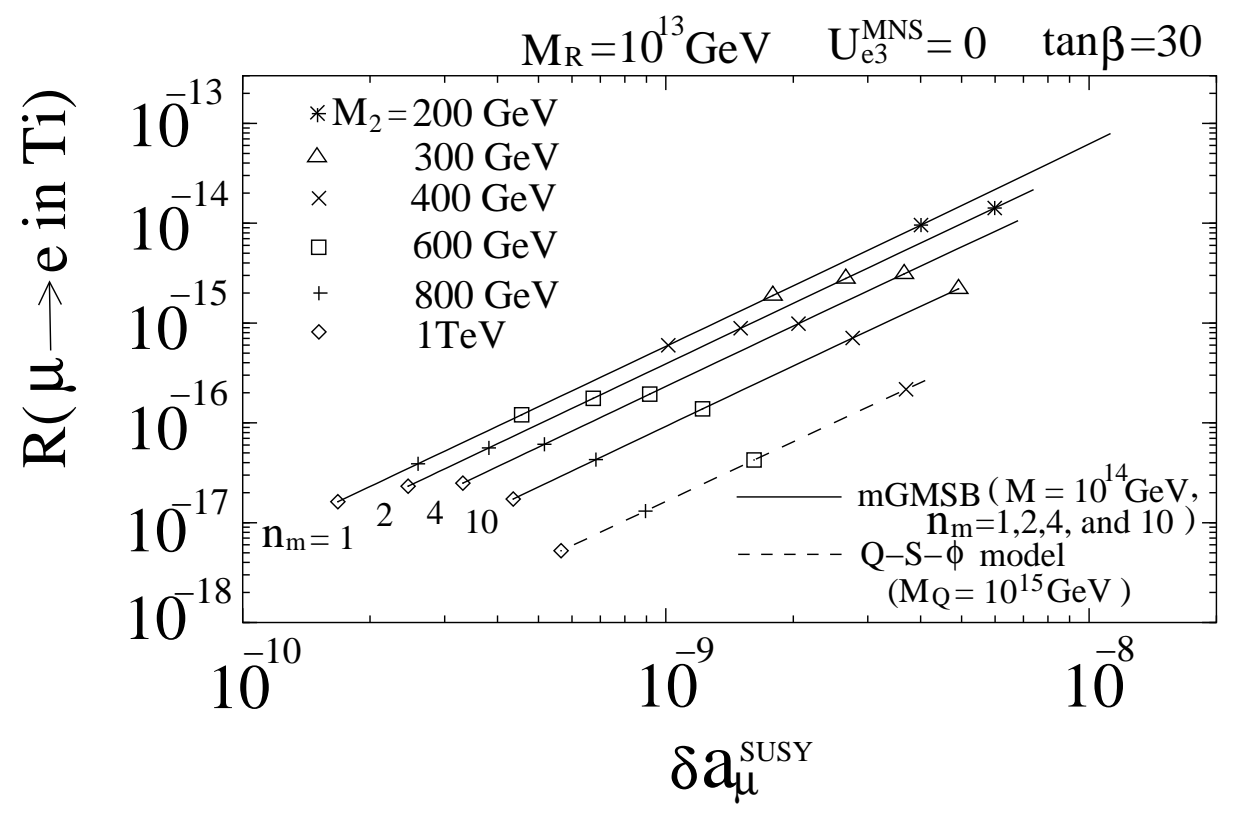

Figure 2: Relations between $R(\mu \rightarrow e)$ and superpartner contribution to muon $g-2\left(\delta a_{\mu}^{\mathrm{SUSY}}\right)$ as a function of low-energy wino mass $M_{2}$ in mGMSB with $n_{m}=1,2,4,10$ and in $Q$ - $S$ - $\phi$ model. We assume that $M_{R}=10^{13} \mathrm{GeV}, U_{e 3}^{\mathrm{MNS}}=0, \tan \beta=30$, and the messenger scale $M=10^{14} \mathrm{GeV}$ for mGMSB and $M_{Q}=10^{15} \mathrm{GeV}$ for $Q-S-\phi$ model.

the region around $M_{R}>10^{12-13} \mathrm{GeV}$ and $M>10^{13-14} \mathrm{GeV}$. It is extremely interesting to see from Fig. 1 that future measurements by experiments such as PRIME may be able to reach the region above $M_{R}>10^{11} \mathrm{GeV}$ and $M>10^{12} \mathrm{GeV}$. When one considers that this encompasses the range of allowed messenger mass from the leptogenesis considerations, Eq. (41), the result indicates that LFV signals are expected to be measured provided $M_{R}<M$.

In Fig. 2, we show relations between $R(\mu \rightarrow e)$ and superpartner contribution to muon $g-2\left(\delta a_{\mu}^{\mathrm{SUSY}}\right)$ as a function of the low-energy wino mass $M_{2}$ in $\mathrm{mGMSB}$ with $n_{m}=1,2,4,10$ and in the $Q-S$ - $\phi$ model. Here we have assumed that $M_{R}=10^{13} \mathrm{GeV}, U_{e 3}^{\mathrm{MNS}}=0, \tan \beta=30$, and the messenger scale $M=10^{14} \mathrm{GeV}$ for mGMSB and $M_{Q}=10^{15} \mathrm{GeV}$ for $Q-S$ - $\phi$ model. Note that a recent updated estimate of muon $g-2[28$, shows that the deviations of the SM prediction from the measurement at BNL [29] are found to be

$$
\delta a_{\mu}=\left\{\begin{array}{c}
(22.1 \pm 7.2 \pm 3.5 \pm 8.0) \times 10^{-10} \quad\left[e^{+} e^{-}-\text {based estimate }\right] \\
(7.4 \pm 5.8 \pm 3.5 \pm 8.0) \times 10^{-10} \quad[\tau-\text { based estimate }]
\end{array}\right.
$$

where the first error comes from the hadronic contribution, the second one from the light-bylight scattering contribution and the third one from the BNL experiment. For fixed $M_{2}$, as 
the number of messenger multiplets $n_{m}$ gets larger, $\delta a_{\mu}^{\mathrm{SUSY}}$ increases because slepton masses become smaller (see Eq. (20) $)$, on the other hand, $R(\mu \rightarrow e$ ) does not change much because the smaller slepton masses also slightly suppress the LFV masses compared to the diagonal components that originated partially from gaugino masses in the RGE running. In the $Q-S-\phi$ model, relatively large scalar masses, which are flavor diagonal, are additionally generated at $S$ and $\phi$ mass thresholds, which are below the right-handed neutrino mass scale. Therefore the LFV masses are relatively suppressed, and as a result, the conversion rate gets smaller. Taking into account the allowed range of $M_{Q}\left(10^{14} \mathrm{GeV}<M_{Q}<10^{16} \mathrm{GeV}\right)$ [13, however, the present and future LFV searches have great impact on sensitivity to this model too.

LFV event rates depend on parameters in the neutrino sector such as $M_{R}$ and $U_{e 3}^{\mathrm{MNS}}$ as shown in Fig. 1. On the other hand, the contribution from superpartners to muon $g-2$ is almost independent of these parameters because small LFV slepton masses do not affect it. So in the parameter space in Fig. 1] the superpartner contribution to muon $g-2$ is almost constant, $\delta a_{\mu}^{\text {SUSY }} \simeq 2 \times 10^{-9}$. For fixed $\delta a_{\mu}^{\text {SUSY }}$, the messenger-model dependence can be read from Fig. 2] and the dependence on the neutrino parameters and the messenger scale can be seen from Fig. 1. It should be noted also that $\delta a_{\mu}^{\text {SUSY }}$ is approximately proportional to tan $\beta$, and $R(\mu \rightarrow e)$ is proportional to $\tan ^{2} \beta$. Since $R(\mu \rightarrow e) \propto\left(\delta a_{\mu}^{\mathrm{SUSY}}\right)^{2}$ as can be seen from Fig. 2 [30, potentially the precise determination of muon $g-2$ together with LFV searches would provide a significant handle on models with LFV.

\subsection{Non-degenerate right-handed neutrinos $M_{R_{3}} \geq M_{R_{2}} \geq M_{R_{1}}$}

When the right-handed neutrinos are not degenerate $M_{R_{3}} \geq M_{R_{2}} \geq M_{R_{1}}$, a dependence on the $R$-matrix enters in the slepton masses, and hence the LFV event rates generally depend on the unknown ${ }^{8}$ parameters of $R_{i j}$.

First let us consider the case with $R=\mathbf{1}$. When $U_{e 3}^{\mathrm{MNS}}=0$, the third generation righthanded neutrino does not induce $\mu-e$ flavor violation. In this case, whatever the value of $M_{R_{3}}$ is (even if $M_{R_{3}}>M$ ), the $\mu-e$ conversion rate is the same as that found in Fig. 1 except that now we should read $M_{R}$ in Fig. 11 as $M_{R_{2}}$. However, LFV generated by the third generation right-handed neutrino is important in the $\tau \rightarrow \mu \gamma$ process especially when $M>M_{R_{3}}$ and $M_{R_{2}}$ is rather small (say, less than $10^{12} \mathrm{GeV}$ ). In Fig. 3, branching ratio contours of $\tau \rightarrow \mu \gamma$ are shown as a function of the messenger scale $M$ and the third

\footnotetext{
${ }^{8}$ None of the successful neutrino oscillation experiments can pin down or even constrain the $R$-matrix entries. It takes additional observables, such as LFV signals, to make progress in constraining this matrix.
} 


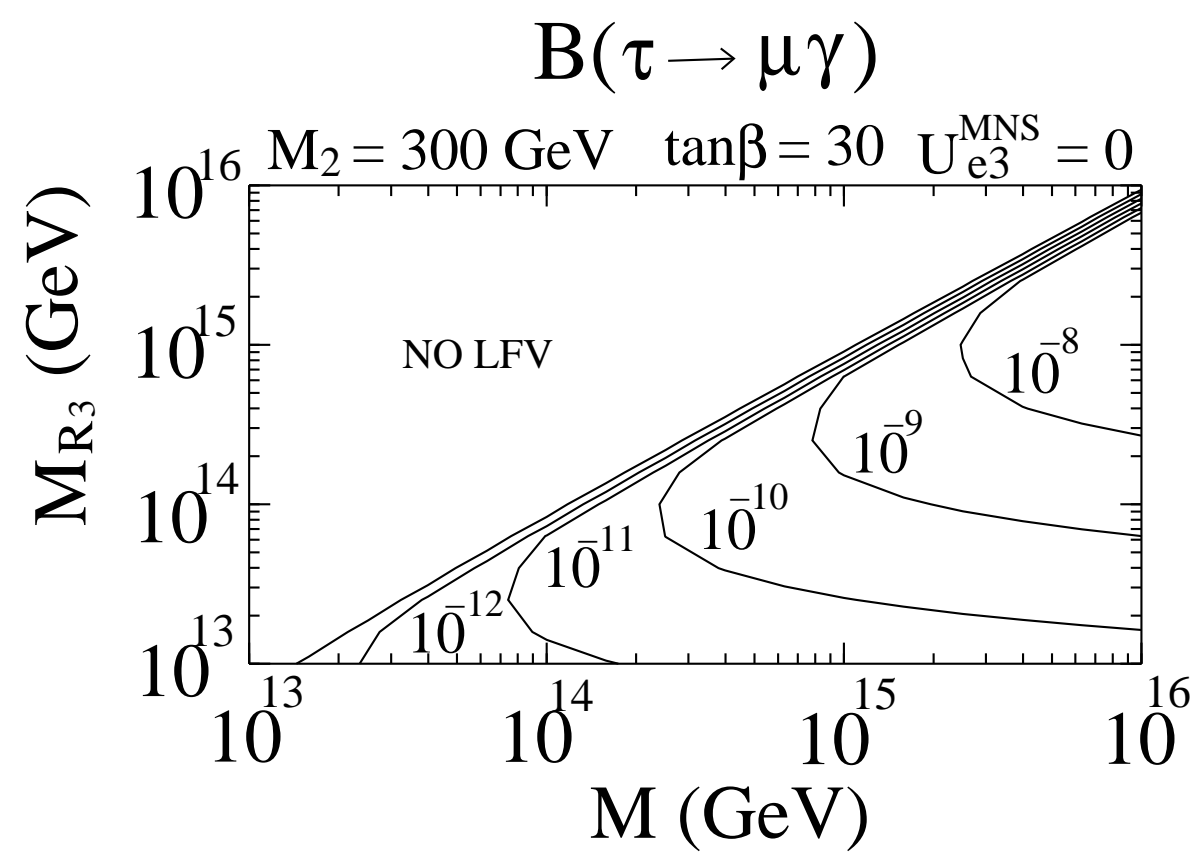

Figure 3: Branching ratios of $\tau \rightarrow \mu \gamma$ process, $B(\tau \rightarrow \mu \gamma)$ as a function of the messenger scale $M$ and the third generation right-handed neutrino mass $M_{R_{3}}$. Here we consider the hierarchical right-handed neutrinos, $M_{R_{1}}=10^{10} \mathrm{GeV}$ and $M_{R_{2}}=10^{12} \mathrm{GeV}$. We assume mGMSB with $n_{m}=1$ and $M_{2}=300 \mathrm{GeV}$, and $\tan \beta=30, U_{e 3}^{\mathrm{MNS}}=0$ and $R=1$. Future experimental sensitivity might be as low as $10^{-9}$, in which case there is a significant region of $M_{R_{3}}$ and $M$ that this LFV observable would probe.

generation right-handed neutrino mass $M_{R_{3}}$. Here we assumed mGMSB with $n_{m}=1$ and $M_{2}=300 \mathrm{GeV}, \tan \beta=30, U_{e 3}^{\mathrm{MNS}}=0$ and $R=1$. We took $M_{R_{1}}=10^{10} \mathrm{GeV}, M_{R_{2}}=10^{12}$ $\mathrm{GeV}$, and thus the $\mu-e$ conversion is typically small $\left(R(\mu \rightarrow e) \sim 10^{-16}-10^{-17}\right)$ because the third generation right-handed neutrino does not contribute $\left(U_{e 3}^{\mathrm{MNS}}=0\right)$ and $M_{R_{2}}$ is so low. Nevertheless, as can be seen from Fig. B. the search for $\tau \rightarrow \mu \gamma$ could be interesting and capable of probing the region around $M>10^{15} \mathrm{GeV}$ and $M_{R_{3}}>10^{14} \mathrm{GeV}$ if the future improved measurement of $B(\tau \rightarrow \mu \gamma)$ gets to the level of $10^{-8}-10^{-9}$ [31]. Therefore $\tau \rightarrow \mu \gamma$ will be independently important in the framework of GMSB.

Next, we consider cases where $R \neq \mathbf{1}$. We parameterize the $R$ matrix as follows:

$$
R=\left(\begin{array}{ccc}
c_{R_{2}} c_{R_{1}} & s_{R_{2}} c_{R_{1}} & s_{R_{1}} \\
-s_{R_{2}} c_{R_{3}}-c_{R_{2}} s_{R_{3}} s_{R_{1}} & c_{R_{2}} c_{R_{3}}-s_{R_{2}} s_{R_{3}} s_{R_{1}} & s_{R_{3}} c_{R_{1}} \\
s_{R_{2}} s_{R_{3}}-c_{R_{2}} c_{R_{3}} s_{R_{1}} & -c_{R_{2}} s_{R_{3}}-s_{R_{2}} c_{R_{3}} s_{R_{2}} & c_{R_{3}} c_{R_{1}}
\end{array}\right),
$$

where $s_{R i}=\sin \theta_{R_{i}}$ and $c_{R i}=\cos \theta_{R_{i}}$. 


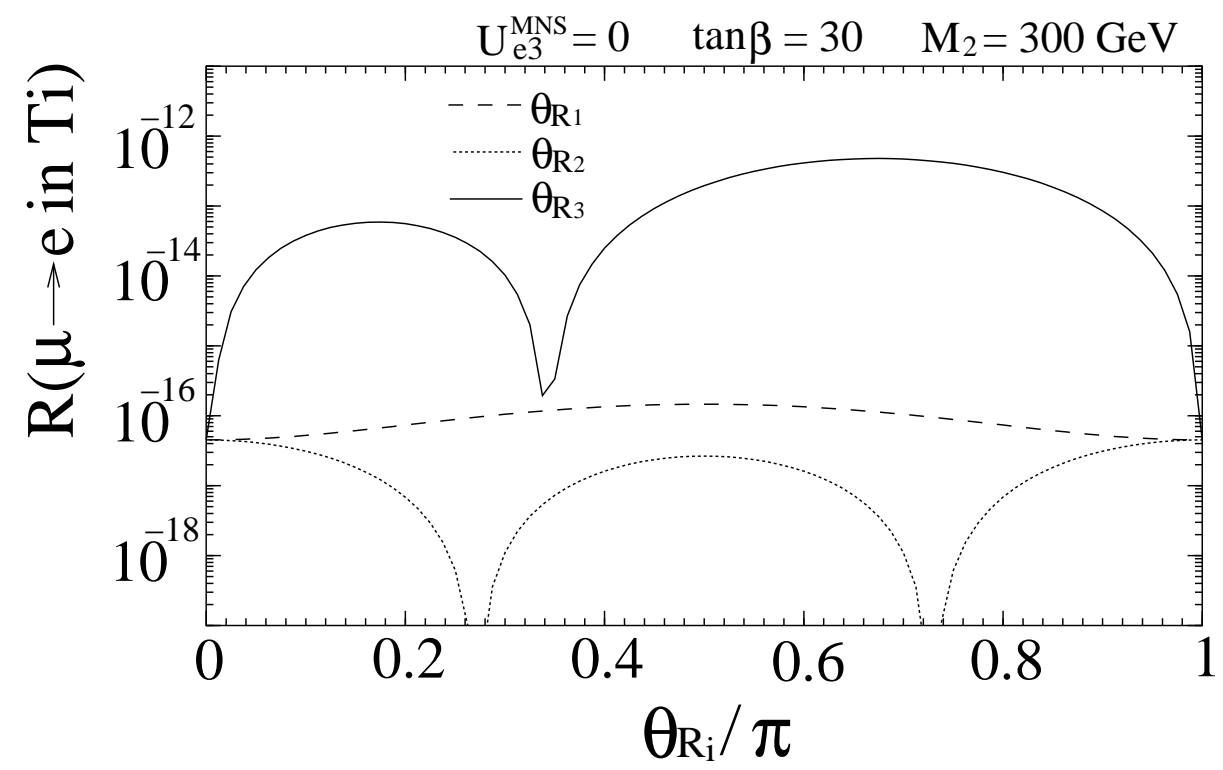

Figure 4: The $\mu-e$ conversion rates, $R\left(\mu \rightarrow e\right.$ in Ti), as a function of an angle $\theta_{R_{i}}$ in the unknown $R$-matrix. Each line in the plot is drawn by varying only one non-zero angle $\theta_{R_{i}}$. We assume mGMSB with $n_{m}=1$ and $M_{2}=300 \mathrm{GeV}, \tan \beta=30$ and $U_{e 3}^{\mathrm{MNS}}=0$. Here we set $M=10^{15} \mathrm{GeV}, M_{R_{1}}=10^{10} \mathrm{GeV}, M_{R_{2}}=10^{12} \mathrm{GeV}$, and $M_{R_{3}}=10^{14} \mathrm{GeV}$.

Assuming that one of the angles $\theta_{R_{i}}$ is non-zero, we present $\mu-e$ conversion rates showing the dependence on each angle $\theta_{R_{i}}$ in Fig. 团 and 15 . We assumed mGMSB with $n_{m}=1$, $M_{2}=300 \mathrm{GeV}$ and $\tan \beta=30$. We fixed the messenger scale and right-handed neutrino masses to be $M=10^{15} \mathrm{GeV}, M_{R_{1}}=10^{10} \mathrm{GeV}, M_{R_{2}}=10^{12} \mathrm{GeV}$, and $M_{R_{3}}=10^{14} \mathrm{GeV}$. In Fig. (4. $U_{e 3}^{\mathrm{MNS}}$ is set to zero. As can be understood from Eq. (19), due to non-zero mixing in the $R$-matrix, several comparable terms are generated in Eq. (19), and then for some specific values of $\theta_{R_{i}}$, a cancellation occurs between terms. That is why we find some steep suppressions of $R(\mu \rightarrow e)$ in Fig. [ Otherwise the event rates in most of Fig. 团 are comparable or larger than those when $R=\mathbf{1}\left(\theta_{R_{i}}=0\right)$.

In Fig. [5, we show a similar figure to Fig. 团except that $U_{e 3}^{\mathrm{MNS}}$ is set to be 0.1. Again at some (different) values of $\theta_{R_{i}}$ a cancellation occurs and $R(\mu-e)$ dips precipitously. However, the typical value of the event rates are often considerably larger than those when $U_{e 3}^{\mathrm{MNS}}=0$, especially when the hierarchy between $M_{R_{2}}$ and $M_{R_{3}}$ is large. Therefore, we conclude that we should generally expect large LFV event rates even though the $R$-matrix is unknown. 


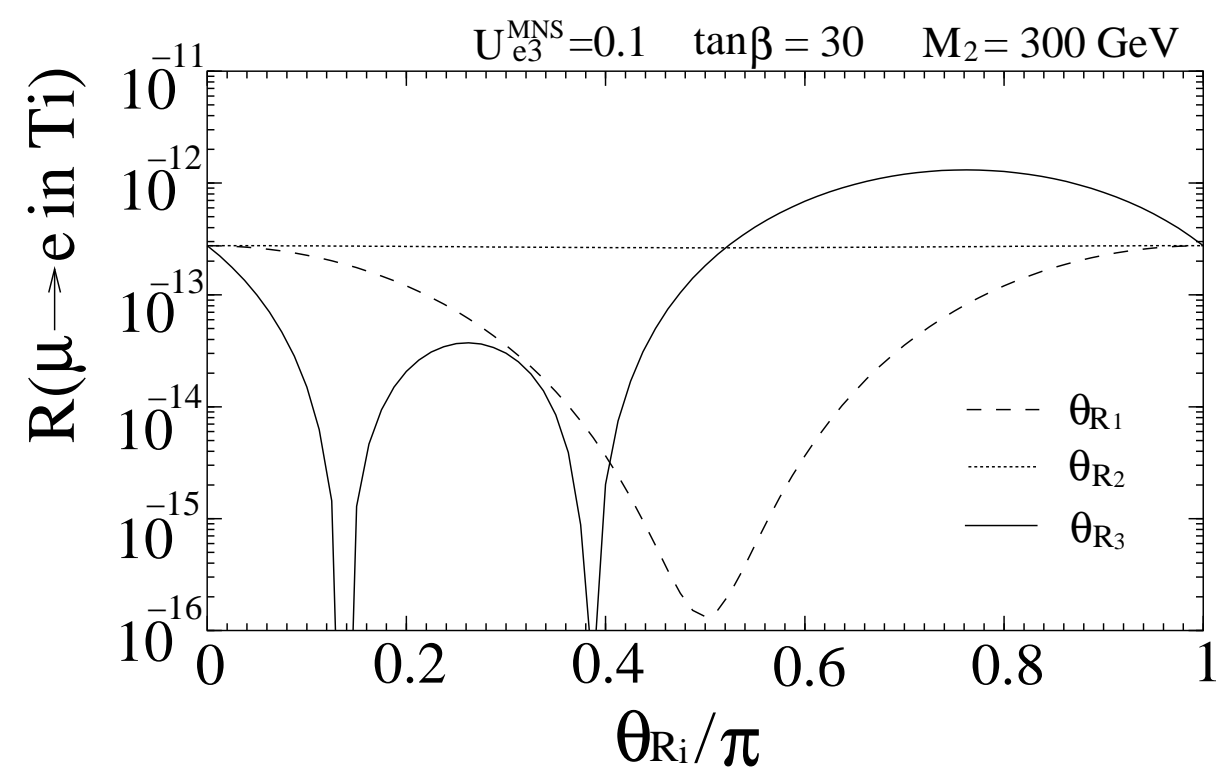

Figure 5: Same as Fig. 团 except for $U_{e 3}^{\mathrm{MNS}}=0.1$.

\section{Conclusion}

We have found that if we incorporate neutrino masses and leptogenesis into the superstructure of direct gauge-mediated supersymmetry breaking, significant low-energy LFV signals are possible in observables such as $\mu \rightarrow e \gamma, \mu-e$ conversion in nuclei and $\tau \rightarrow \mu \gamma$. Heavy right-handed neutrinos below the high messenger scale are what induce the large rates. This is because the full Yukawa couplings of neutrinos are part of the effective theory between the messenger scale and the lower right-handed neutrino scale, thereby enabling their highly mixed off-diagonal entries to infect the slepton masses via renormalization group running. The large LFV mass entries in the slepton mass matrix that result from the neutrino Yukawa coupling RG-effect contribute in loops to the LFV observables.

We have shown that small regions of parameter space are already ruled out by carefully correlating what the theories predict for LFV observables to what the experiments have (not) measured. Large regions of parameter space will be tested and probed by future experiments dedicated to significant improvements in the search for $\mu \rightarrow e \gamma, \mu-e$ conversion in nuclei and $\tau \rightarrow \mu \gamma$. And as we have emphasized in this article, a non-zero measurement of these LFV decays is not only consistent with some versions of gauge mediation, it is to be expected. 


\section{Acknowledgements}

This work was supported in part by the Department of Energy and the Alfred P. Sloan Foundation.

\section{References}

[1] M. Dine, A. E. Nelson and Y. Shirman, "Low-energy dynamical supersymmetry breaking simplified," Phys. Rev. D 51, 1362 (1995) hep-ph/9408384; M. Dine, A. E. Nelson, Y. Nir and Y. Shirman, "New tools for low-energy dynamical supersymmetry breaking," Phys. Rev. D 53, 2658 (1996) hep-ph/9507378.

[2] G. F. Giudice and R. Rattazzi, "Theories with gauge-mediated supersymmetry breaking," Phys. Rept. 322, 419 (1999) hep-ph/9801271.

[3] T. Yanagida, in Proc. Workshop on the unified theory and the baryon number in the universe, (Tsukuba, 1979), eds. O. Sawada and S. Sugamoto, Report KEK-79-18 (1979); S.L. Glashow, in Quarks and Leptons, (Cargése, 1979), eds. M. Lèvy et al. (Plenum, 1980, New York); M. Gell-Mann, P. Ramond and R. Slansky, in Supergravity, (NorthHolland, Amsterdam, 1979), eds. D.Z. Freedman and P. van Nieuwenhuizen.

[4] M. Fukugita and T. Yanagida, "Baryogenesis Without Grand Unification," Phys. Lett. B 174, 45 (1986).

[5] T. Asaka, K. Hamaguchi, M. Kawasaki and T. Yanagida, "Leptogenesis in inflaton decay," Phys. Lett. B 464, 12 (1999) hep-ph/9906366; T. Asaka, K. Hamaguchi, M. Kawasaki and T. Yanagida, "Leptogenesis in inflationary universe," Phys. Rev. D 61, 083512 (2000) hep-ph/9907559.

[6] K. Hamaguchi, H. Murayama and T. Yanagida, "Leptogenesis from $\tilde{N}$-dominated early universe," Phys. Rev. D 65, 043512 (2002) hep-ph/0109030.

[7] T. Moroi, "Effects of the gravitino on the inflationary universe," hep-ph/9503210.

[8] M. Fujii and T. Yanagida, "Natural gravitino dark matter and thermal leptogenesis in gauge-mediated supersymmetry-breaking models," Phys. Lett. B 549, 273 (2002) hep-ph/0208191; M. Fujii, M. Ibe and T. Yanagida, "Thermal leptogenesis and gauge mediation," hep-ph/0309064. 
[9] K. I. Izawa and T. Yanagida, Prog. Theor. Phys. 95, 829 (1996) hep-th/9602180.

[10] K. A. Intriligator and S. Thomas, "Dynamical Supersymmetry Breaking on Quantum Moduli Spaces," Nucl. Phys. B 473, 121 (1996) hep-th/9603158.

[11] N. Arkani-Hamed, J. March-Russell and H. Murayama, "Building models of gaugemediated supersymmetry breaking without a messenger sector," Nucl. Phys. B 509, 3 (1998) hep-ph/9701286.

[12] S. Dimopoulos, G. R. Dvali, R. Rattazzi and G. F. Giudice, "Dynamical soft terms with unbroken supersymmetry," Nucl. Phys. B 510, 12 (1998) hep-ph/9705307.

[13] H. Murayama, "A model of direct gauge mediation," Phys. Rev. Lett. 79, 18 (1997) hep-ph/9705271.

[14] K. I. Izawa, Y. Nomura, K. Tobe and T. Yanagida, "Direct-transmission models of dynamical supersymmetry breaking," Phys. Rev. D 56, 2886 (1997) hep-ph/9705228.

[15] J. R. Ellis and M. Raidal, "Leptogenesis and the violation of lepton number and CP at low energies," Nucl. Phys. B 643, 229 (2002) hep-ph/0206174; J. R. Ellis, M. Raidal and T. Yanagida, "Sneutrino inflation in the light of WMAP: Reheating, leptogenesis and flavor-violating lepton decays," hep-ph/0303242.

[16] Z. Maki, M. Nakagawa and S. Sakata, "Remarks On The Unified Model Of Elementary Particles," Prog. Theor. Phys. 28, 870 (1962).

[17] J. A. Casas and A. Ibarra, Nucl. Phys. B 618, 171 (2001) hep-ph/0103065.

[18] Koichiro Nishikawa, "Results and status of current accelerator neutrino experiments," Lepton photon 2003, http://conferences.fnal.gov/lp2003/program/index.html.

[19] S. N. Ahmed et al. [SNO Collaboration], "Measurement of the total active B-8 solar neutrino flux at the Sudbury Neutrino Observatory with enhanced neutral current sensitivity," nucl-ex/0309004. See also a recent Super-Kamiokande result: M.B. Smy et al. [Super-Kamiokande Collaboration], "Precise measurement of the solar neutrino day/night and seasonal variation in Super-Kamiokande-I," hep-ex/0309011.

[20] F. Borzumati and A. Masiero, "Large Muon And Electron Number Violations In Supergravity Theories," Phys. Rev. Lett. 57, 961 (1986). 
[21] J. Hisano, T. Moroi, K. Tobe, M. Yamaguchi and T. Yanagida, "Lepton flavor violation in the supersymmetric standard model with seesaw induced neutrino masses," Phys. Lett. B 357, 579 (1995) hep-ph/9501407; J. Hisano, T. Moroi, K. Tobe and M. Yamaguchi, "Lepton-Flavor Violation via Right-Handed Neutrino Yukawa Couplings in Supersymmetric Standard Model," Phys. Rev. D 53, 2442 (1996) hep-ph/9510309.

[22] G. F. Giudice and R. Rattazzi, "Extracting supersymmetry-breaking effects from wavefunction renormalization," Nucl. Phys. B 511, 25 (1998) hep-ph/9706540.

[23] R. Barbieri and L. J. Hall, "Signals for supersymmetric unification," Phys. Lett. B 338, 212 (1994) hep-ph/9408406.

[24] For update on the expected sensitivity of MEG experiment, see MEG home page, http://meg.icepp.s.u-tokyo.ac.jp.

[25] For a summary of current limits and future prospects of $\mu-e$ conversion and $\mu \rightarrow e \gamma$ experiments, see Michael Hebert, "Status and Perspectives of MECO, the Muon to Electron Conversion Experiment," Presented at Beyond the Desert 2003, Schloss Ringberg, June 9-14, 2003, http://meco.ps.uci.edu/mecopages/presentations.html.

[26] See MECO experiment home page, http://meco.ps.uci.edu.

[27] For future possibility of $\mu-e$ conversion experiment, see CERN study, J. Aysto et al., hep-ph/0109217. See also a letter of intent to the J-PARC experiment (L25) (PRIME experiment), http://www-ps.kek.jp/jhf-np/index_e.html.

[28] M. Davier, S. Eidelman, A. Hocker and Z. Zhang, "Updated estimate of the muon magnetic moment using revised results from $e^{+} e^{-}$annihilation," hep-ph/0308213. See also more conservative approach to interpret the muon $g-2$ results, S. P. Martin and J. D. Wells, "Super-conservative interpretation of muon $g-2$ results applied to supersymmetry," Phys. Rev. D 67, 015002 (2003) hep-ph/0209309.

[29] G. W. Bennett et al. [Muon $g-2$ Collaboration], "Measurement of the positive muon anomalous magnetic moment to 0.7-ppm," Phys. Rev. Lett. 89, 101804 (2002) [Erratumibid. 89, 129903 (2002)] hep-ex/0208001.

[30] J. Hisano and K. Tobe, "Neutrino masses, muon $g-2$, and lepton-flavour violation in the supersymmetric see-saw model," Phys. Lett. B 510, 197 (2001) hep-ph/0102315. 
[31] T. Oshima, " $\tau \rightarrow \mu \gamma$ : Status and prospects," 3rd Workshop on Neutrino Oscillations and Their Origin (NOON 2001), Kashiwa, Japan, 5-8 Dec 2001; K. Inami, "Lepton flavor violation in tau decay: experiment," Mini-workshop for Super KEKB LoI physics part Discovery and Identification of New Physics at $3 a b^{-1}$ and $30 a b^{-1}$, KEK, 19 June 2003. 